Trauma Berufskrankh 2018 20 (Suppl 3):S171-S176 https://doi.org/10.1007/s10039-018-0359-x Online publiziert: 6. März 2018

(c) Springer Medizin Verlag $\mathrm{GmbH}$, ein Teil von Springer Nature 2018

CrossMark
I. A. Ederer · A. Nusche - A. Daigeler · C. Bösch

Klinik für Hand-, Plastische, Rekonstruktive und Verbrennungschirurgie, BG Klinik Tübingen, Tübingen, Deutschland

\section{Versorgung von frischen Beugesehnenverletzungen}

Handverletzungen [9]. Angesichts der hohen Anzahl von Handverletzungen in der Primärversorgung ist dies eine nicht zu unterschätzende Zahl mit bedeutsamen sozioökonomischen Folgen, da wegen der Nachbehandlungszeit von etwa 3 Monaten ein entsprechender Ausfall der beruflichen Tätigkeit resultiert.

\section{Anatomie}

Die anatomischen Kenntnisse des Beugesehnenapparats stellen eine unabdingbare Grundlage für die Diagnostik dar. Prinzipiell unterscheidet man zwischen dem extrinsischen und intrinsischen Beugesystem. Die extrinsische Muskulatur wird weiterführend in die oberflächlichen und tiefen Beugesehnen (M. flexor digitorum superficialis et profundus [FDS et FDP]) unterteilt. Die einzige extrinsische Beugesehne des Daumens ist die Sehne des M. flexor pollicis longus (FPL). Die Namensgebung in oberflächlich und tief ist nur bedingt topografisch einwandfrei, da die tiefe Beugesehne auf Höhe des proximalen Grundgliedes durch das Chiasma der oberflächlichen Beugesehne hindurchtritt, um an der Endgliedbasis des jeweiligen Fingers zu inserieren (• Abb. 1). So ergibt sich eine oberflächliche Lage der tiefen Beugesehne ab Grundgliedmitte. Die FDS hingegen inseriert mit einem ulnaren und radialen Zügel auf Höhe der Mittelgliedbasis. Nahe der Ansatzstelle der Beugesehnen am jeweiligen Finger finden sich Vincula, die Blutgefäße enthalten, die die Beugesehnen von dorsal versorgen.

Zur intrinsischen Muskulatur zählen unter anderem die Mm. lumbricales et interossei, die ihren Ansatz an der Dorsalaponeurose der jeweiligen Finger finden und daher nur eine Beugung in den Metakarpophalangealgelenken bei Streckung der Finger in den Mittel- und Endgelenken bewirken. Aufgrund ihrer tiefen Lage sind diese nur bei ausgeprägten Verletzungen der Hand betroffen.

Die Sehnen der extrinsischen Muskulatur verlaufen am Finger in einer gemeinsamen Sehnenscheide (Vagina synovialis digitorum manus), die ein reibungsloses Gleiten ermöglicht. Durch die Bewegung der Sehnen in dieser entsteht ein Pumpeffekt, der Synovia in die Sehne transportiert und zur Ernährung des Sehnengewebes beiträgt. An den äußeren Hüllen der Sehnenscheide setzen ligamentäre Strukturen Ringbänder (Ligg. anularia) und Kreuzbänder (Ligg. obliqua) - an, die ein palmares Abweichen der Sehnen bei Flexion verhindern.

\section{Zoneneinteilung}

Entsprechend der Verletzungslokalisation werden unterschiedliche therapeutische Optionen beschrieben [8, 9]. Die topografische Einteilung in 5 Zonen (- Abb. 2), beruht auf den Untersuchungen von Verdan [26], wobei die Zonen am Daumen durch den Buchstaben „T“ gekennzeichnet werden.

\section{Verletzungsursachen}

Bei jeder Anamneseerhebung ist es essenziell, den Verletzungsmechanismus und die Art der Verletzung zu beschreiben, um mögliche Begleitverletzungen zu erfassen und ggf. eine weitere Diagnostik zu veranlassen. Auch aus versicherungsrechtlicher Sicht ist es - v. a. bei Arbeits-
[7]. Insgesamt beträgt die Inzidenz von Beugesehnenverletzungen etwa $1 \%$ aller 


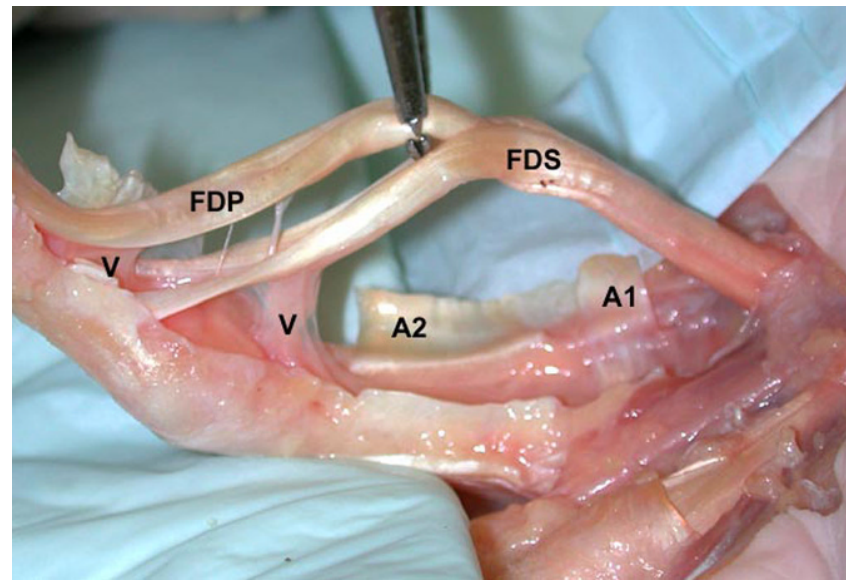

Abb. $1<$ Anatomie der Beugesehnen auf Höhe des Chiasma tendineum. $V$ Vincula, A1/A2 Ringbänder, FDP Flexor digitorum profundus, FDS Flexor digitorum superficialis. (Mit freundl. Genehmigung zur Verfügung gestellt von [18])

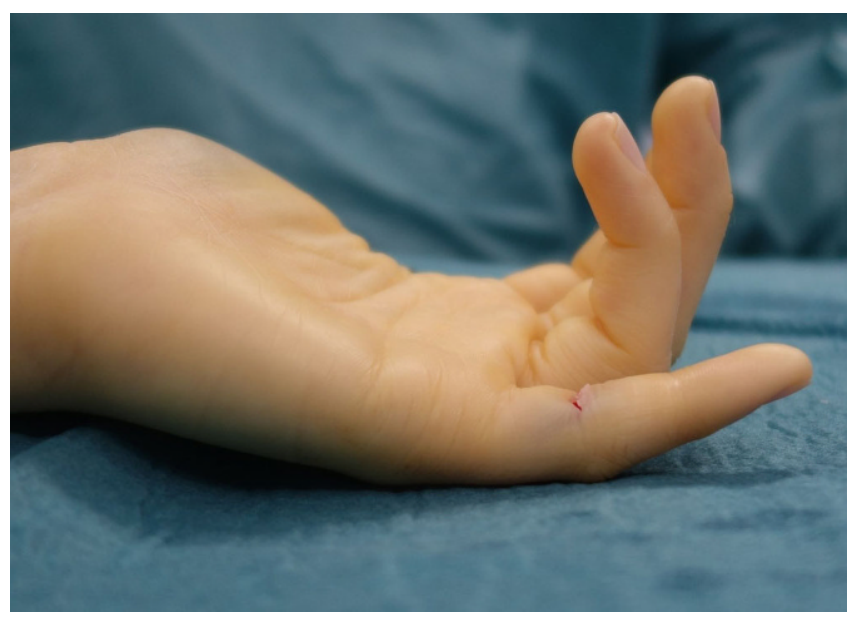

Abb. $3<$ Tonusveränderung am Kleinfinger als Hinweis für eine Beugesehnenverletzung

unfällen - wichtig, den genauen Unfallhergang zu dokumentieren.

Grundsätzlich wird zwischen offenen und gedeckten Sehnenverletzungen unterschieden. In der Regel sind scharfe oder spitze Gegenstände jeglicher Art (Glassplitter, Sägen, Messer, scharfe Metallkanten etc.) Ursachen einer Beugesehnenverletzung [7]. Bei Schnittverletzungen ist stets darauf zu achten, in welcher Fingerposition die Gewalteinwirkung erfolgte: findet die Sehnendurchtrennung bei gebeugtem Finger statt, rutscht der körperferne Stumpf bei nachfolgender Streckung vom sichtbaren Verletzungsort weiter nach distal [11]. Bei Verletzungen am gestreckten Finger hingegen liegt der distale Stumpf nahe der Wunde, wobei häufig der proximale Anteil durch den Muskeltonus bis in die Hohlhand zurückgleiten kann. Die Kenntnis des Unfallmechanismus beeinflusst also die Schnitterweiterung.
Geschlossene Beugesehnenverletzungen sind vergleichsweise selten und zumeist durch eine unerwartete große Krafteinwirkung bedingt $[9,11]$. Im Extremfall kommt es zum knöchernen Ausriss der tiefen Beugesehne aus der Endgliedbasis. Diese Entität ist rar, tritt jedoch am häufigsten beim Sport auf und wird auch als "Rugby-Finger" bezeichnet. Prozentual gesehen betrifft dieses Verletzungsmuster zu rund $80 \%$ den Ringfinger [9].

Beugesehnenverletzungen ohne adäquates Trauma können infolge bestehender Erkrankungen aus dem rheumatischen Formenkreis durch synovialitische Veränderungen bedingt sein [5]. Scharfkantige Knochenvorsprünge können zudem die Ruptur der entzündlich veränderten Sehne bewirken, wovon v.a. die lange Daumenbeugesehne betroffen ist (Mannerfelt-Syndrom; [5]). Degenerative Knochenveränderungen, etwa arthrotische Exophyten im Karpal-

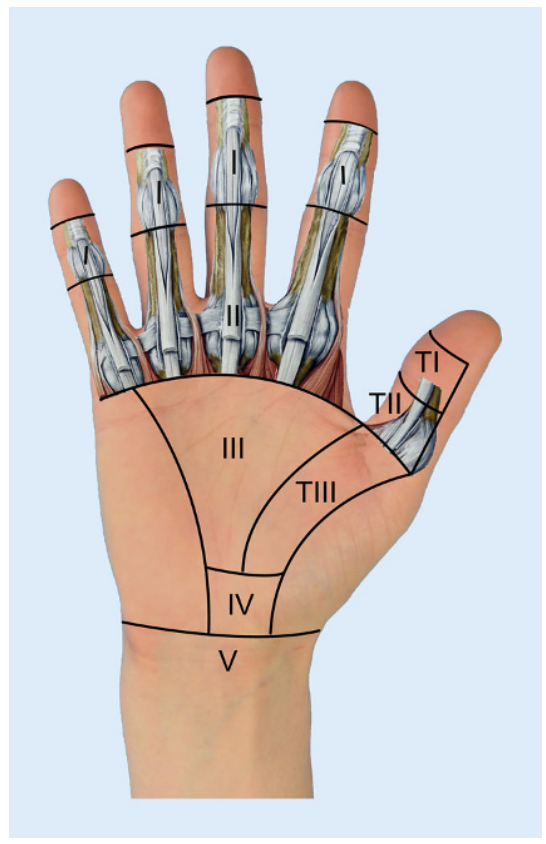

Abb. 2 \ Zoneneinteilung der Beugesehnenverletzungen. (Adaptiert aus [17]; mit freundl. Genehmigung des Thieme Verlags)

kanal, und Morbus Kienböck sind als weitere Ursachen von Sehnenrupturen $\mathrm{zu}$ nennen $[14,16]$. Auch einliegendes Osteosynthesematerial, wie z. B. bei palmar versorgter distaler Radiusfraktur, kann durch mechanische Irritation der darüber liegenden Beugesehnen zu einer gedeckten Verletzung führen $[1,4]$.

\section{Diagnostik}

Bei offenen Verletzungen erlaubt die genaue Lokalisation der Wunde bereits Rückschlüsse auf eine Sehnenbeteiligung. Bei Kleinkindern oder älteren Personen ist eine differenzierte motorische Prüfung oftmals nur erschwert möglich.

Im Ruhezustand zeigt die Hand einen zunehmenden Beugesehnentonus der Finger vom Zeigefinger hin zum Kleinfinger [5]. Jede Abweichung dieses Spannungsverlaufs ist Hinweis auf eine Beugesehnenverletzung (• Abb. 3). Bei komplettem Tonusverlust eines Fingers ist eine kombinierte Durchtrennung beider Beugesehnen anzunehmen. Isolierte Durchtrennungen der FDS hingegen führen nur zu einer minimalen Veränderung der Fingerspannung, da die intakte FDP den Tonus aufrechterhält. 
Folglich ist die Durchführung aktiver und passiver Tests zur Diagnosesicherung unerlässlich.

Die Beugung im Fingerendgelenk wird allein durch die FDP vollzogen. Bei Durchtrennung dieser - egal in welcher Zone - bleibt das Endgelenk beim Faustschluss gestreckt (Winfield-Zeichen; [9]). Die aktive Überprüfung der tiefen Beugesehne erfolgt bei Fixierung des Mittelgliedes und Aufforderung der Patienten, den jeweiligen Finger im Endgelenk zu beugen. Bei Teildurchtrennung der Sehne ist eine aktive Beugung noch möglich; bei Testung gegen Widerstand zeigt sich oftmals eine Schmerzsymptomatik.

Die Funktionsprüfung der FDS ist erheblich schwieriger, da auch die tiefe Beugesehne das Mittelgelenk beugt. Die Testung erfolgt daher nach einem funktionellen Ausschalten der FDP. Hierzu bedient man sich des sog. Quadrigaphänomens: Die benachbarten Finger werden in Überstreckung fixiert und die Patienten zur aktiven Beugung des zu untersuchenden Fingers aufgefordert. Die Beugung im Mittelgelenk erfolgt sodann alleinig durch eine intakte FDS. Am Kleinfinger sind anatomische Varianten der FDS häufig: In etwa $26 \%$ ist diese an die FDS-4 gekoppelt und in bis zu $20 \%$ sogar fehlend [22]. Ein negativer Test muss hier also nicht zwingend auf eine Durchtrennung der Sehne hinweisen. Besondere Aufmerksamkeit verdient auch der Zeigefinger. An diesem ist die FDP häufig nicht gekoppelt mit jener des Mittelfingers und formt in bis zu $20 \%$ einen eigenständigen Muskelbauch, wodurch sie sich ebenso der Fixierung entzieht [28]. So kann durch Beugung des Mittelgelenks eine intakte FDS am Zeigefinger vorgetäuscht werden [9]. Deshalb ist eine Testung der FDS jeden Fingers gegen Widerstand zu empfehlen: Bei Beugung im Mittelgelenk von etwa $90^{\circ}$ und kräftigem Zug am Mittelglied muss das Endgelenk an jedem Finger bei passiver Bewegung widerstandslos und locker beweglich sein. Ist dies nicht der Fall, erfolgt die Beugung über die FDP, die durch den angespannten Tonus die Endgelenkbeweglichkeit einschränkt (•Abb.4).

Bei nichtkooperativen Patienten kann der Tenodeseeffekt hilfreich sein [9]. Da-

Trauma Berufskrankh 2018 · 20 (Suppl 3):S171-S176

https://doi.org/10.1007/s10039-018-0359-x

(c) Springer Medizin Verlag GmbH, ein Teil von Springer Nature 2018

\section{A. Ederer · A. Nusche · A. Daigeler · C. Bösch}

\section{Versorgung von frischen Beugesehnenverletzungen}

\section{Zusammenfassung}

Ziel der chirurgischen Versorgung von Beugesehnenverletzungen ist die frühzeitige Wiederherstellung der Kontinuität durch eine ausreichend stabile Naht, um eine frühfunktionelle passive oder aktive Behandlung zu erlauben und so Adhäsionen der Sehne - insbesondere im osteofibrösen Kanal der Finger - zu vermeiden. In den letzten Jahren wurde eine Vielzahl an Nahttechniken in den unterschiedlichsten Modifikationen beschrieben. Im Sinne "je mehr Nahtmaterial, desto stabiler" wurde der Fokus auf die Erhöhung der Stranganzahl der Kernnaht gelegt, um frühzeitig aktive Nachbehandlungskonzepte zu erlauben. Trotz der höheren initialen biomechanischen Stabilität dieser Nahttechniken in experimentellen Studien konnte in klinischen Übersichtsarbeiten hinsichtlich der Rupturrate kein statistisch signifikanter Unterschied zwischen Zweistrang- und Mehrstrangnähten nachgewiesen werden. Auch bezüglich des funktionellen Ergebnisses war keine Überlegenheit der Mehrstrangtechniken gegenüber den Zweistrangtechniken nachzuweisen. Dementsprechend gibt es bis heute, abgesehen von einer stabilen Kernnaht und einer glättenden Feinadaptation, keinen Goldstandard hinsichtlich der idealen Nahttechnik oder des optimalen Nahtmaterials zur Versorgung von Beugesehnenverletzungen.

Schlüsselwörter

Nahttechnik - Ergebnisse - Zweistrangnaht . Vierstrangnaht $\cdot$ Ruptur

\section{Management of acute flexor tendon injuries}

\section{Abstract}

The aim of acute flexor tendon repair is to establish a strong and stable suture, which enables a smooth gliding of the tendon and early mobilization to prevent postoperative adhesion formation. This is especially important in fingers which have fibrous tendon sheaths. The evolution of different suture techniques in the past decades provides an abundance of surgical options for primary flexor tendon repair. In vitro studies have shown that multistrand core sutures have enhanced biomechanical properties compared to two-strand core sutures. This achievement, however, was not demonstrated in clinical studies as there was no statistically significant difference when looking at the outcomes of rupture rate and function between two-strand and multistrand core sutures. The published literature supports the use of a strong core suture and circumferential epitendinous suture to minimize complications; however, so far there has not been a consensus on the optimal suture technique or material for primary flexor tendon repair.

Keywords

Suture technique - Results - Two-strand suture $\cdot$ Four-strand suture $\cdot$ Rupture runter versteht man eine zunehmende Beugung der Finger bei Streckung im Handgelenk und Streckung der Finger bei Beugung im Handgelenk. Dieser lässt sich durch Druck auf die Muskulatur der entsprechenden Sehnen am Unterarm verstärken. Nach Beugesehnendurchtrennungen ist der Tenodeseeffekt pathologisch. Auch tastbare Unregelmäßigkeiten im Sehnenverlauf bei gleichzeitiger passiver Bewegung des Fingers können auf eine Sehnenverletzung hinweisen.

Jede Handuntersuchung muss auch die Überprüfung der Sensibilität und
Durchblutung beinhalten. Begleitende Nerven- und Gefäßverletzungen sind aufgrund der anatomischen Lagebeziehung nicht selten und unbedingt präoperativ zu erfassen. Zusätzlich sollte ein Röntgenbild in 2 Ebenen angefertigt werden, um Fremdkörper, knöcherne Sehnenausrisse oder Begleitfrakturen auszuschließen. Bei gedeckten Rupturen oder Verdacht auf Ringbandverletzungen ist die Sonographie ein zuverlässiges und meist gut verfügbares diagnostisches Verfahren. 


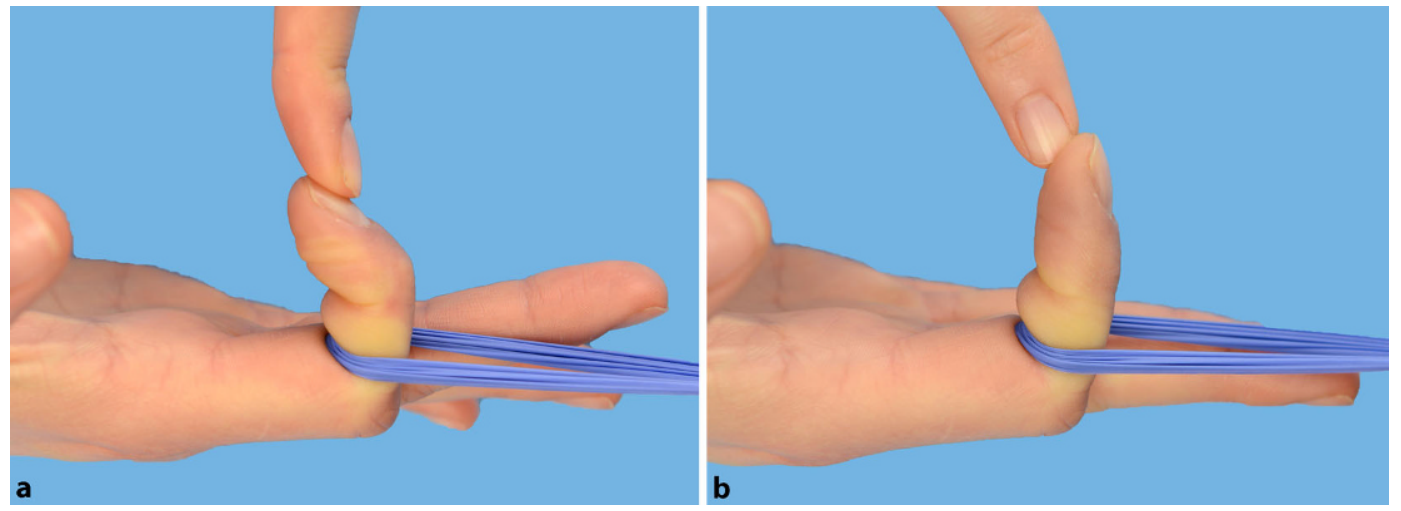

Abb. $4 \triangleleft$ Funktionsüberprüfung der oberflächlichen Beugesehne gegen Widerstand: Das Endgelenk muss bei passiver Flexion (a) und Extension (b) leicht beweglich sein. Ansonsten erfolgt die Beugung im Mittelgelenk durch die Flexor-digitorum-profundus(FDP)-Sehne

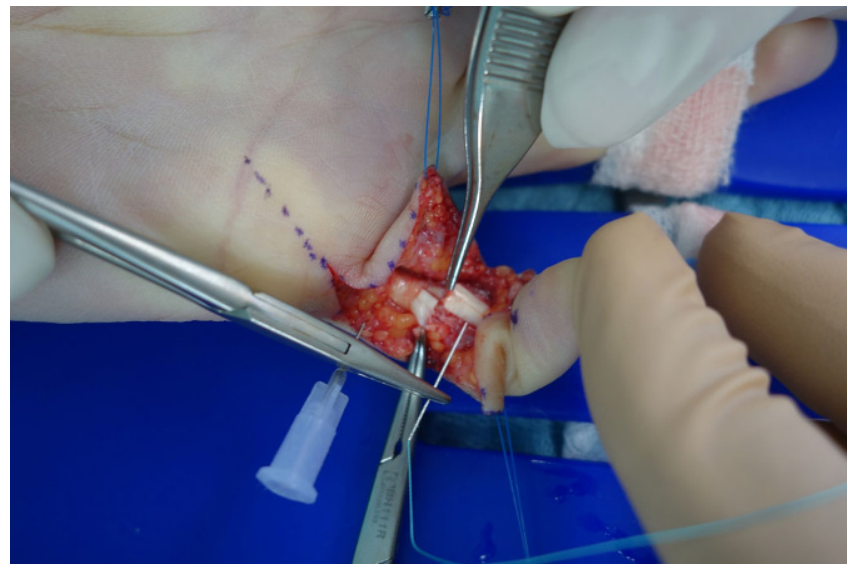

Abb. $5<$ Atraumatisches Fixieren der Beugesehne mit Kanüle vor Beginn der Kernnaht (Schnitterweiterung nach Bruner)

\section{Operative Therapie}

Beugesehnenverletzungen sind möglichst zeitnah nach dem Trauma zu versorgen, um einer Ausbreitung der Keimbesiedlung entgegenzuwirken. Sollte eine primäre Versorgung in den ersten $24 \mathrm{~h}$ nach dem Trauma nicht möglich sein, ist eine spätprimäre Naht innerhalb von 14 Tagen anzustreben [11]. Ein definitiver Zeitpunkt, ab wann eine primäre Naht nicht mehr möglich ist, wird in der Literatur nicht beschrieben. Allerdings können beim Erwachsenen nach der 6. Woche aufgrund von Adhäsionen und der durch die Retraktion der Sehnenstümpfe hervorgerufenen Minderperfusion keine vergleichbaren Ergebnisse mehr erzielt werden [9]. Sehnennähte sollten von Operateuren mit handchirurgischer Erfahrung durchgeführt und im Bedarfsfall an ein Zentrum für Handchirurgie transferiert werden.

Die operative Versorgung erfolgt prinzipiell unter Lupenbrillenvergrößerung und Anlage einer Blutleere. Begleitverletzungen von Gefäßen oder Nerven erfor- dern den zusätzlichen Einsatz des Operationsmikroskops und mikrochirurgischen Instrumentariums.

\section{Schnitterweiterung}

Schräg verlaufende Wunden können in eine Bruner-Schnittführung (• Abb. 5) integriert werden, bis ein Auffinden der Sehnenstümpfe und eine Versorgung von Begleitverletzungen möglich ist [4]. Bei quer über den Finger verlaufenden Verletzungen ist oftmals eine mediolaterale Schnitterweiterung durchzuführen, um einen Hautlappen mit hinreichender Breite zu bekommen. Beim Aufsuchen der Sehnenstümpfe ist auf ein äußerst gewebeschonendes Vorgehen zu achten, um postoperative Adhäsionen zu vermeiden [8]. Distale Sehnenenden lassen sich durch passives Beugen der Finger in die Verletzungszone befördern. Proximale Stümpfe werden durch Beugung im Handgelenk und zusätzliches „Ausmelken“ in die Wunde gebracht. Ist der Sehnenstumpf nicht auffindbar, muss eine Schnitterweiterung oder ein weite- rer Zugang erfolgen. Um ein erneutes Zurückgleiten der Sehnen zu verhindern, können diese temporär mit Kanülen fixiert werden. Ausgefranste Sehnenenden sollten vor der Naht geglättet werden. Es empfiehlt sich, einen Teil der Ausfransung bis zur Vervollständigung der Naht zu belassen, um ebendort die Sehne $\mathrm{zu}$ fassen und somit eine atraumatische Fixierung zu gewährleisten [10]. Hinsichtlich der biomechanischen Stabilität bei Fingerbeugung ist auf eine Schonung der Ringbänder zu achten. Sollten diese die Versorgung oder danach das Gleiten der Sehne behindern, ist eine Verschmälerung, Ringbandplastik oder Aufspaltung zu empfehlen [8]. Einige Studien zeigen sogar, dass selbst die A2und A4-Ringbänder gespalten werden dürfen, sofern die benachbarten Ringbänder in ihrer Kontinuität bestehen bleiben [13, 23, 24].

\section{Nahttechnik}

Ziel der operativen Versorgung von Beugesehnenverletzungen ist eine Naht mit ausreichender Stabilität und guter Gleitfähigkeit, um eine frühfunktionelle passive oder aktive Nachbehandlung zu erlauben. Grundsätzlich besteht die Sehnennaht aus einer Kernnaht und einer epitendinösen Adaptationsnaht.

\section{Kernnaht}

Bezüglich der Vielzahl an Nahttechniken werden sowohl Zweistrang- als auch Mehrstrangtechniken durchgeführt. Obwohl experimentelle Untersuchungen gezeigt hatten, dass Mehrstrangnähte zwar belastbarer sind als Zweistrangnähte, 


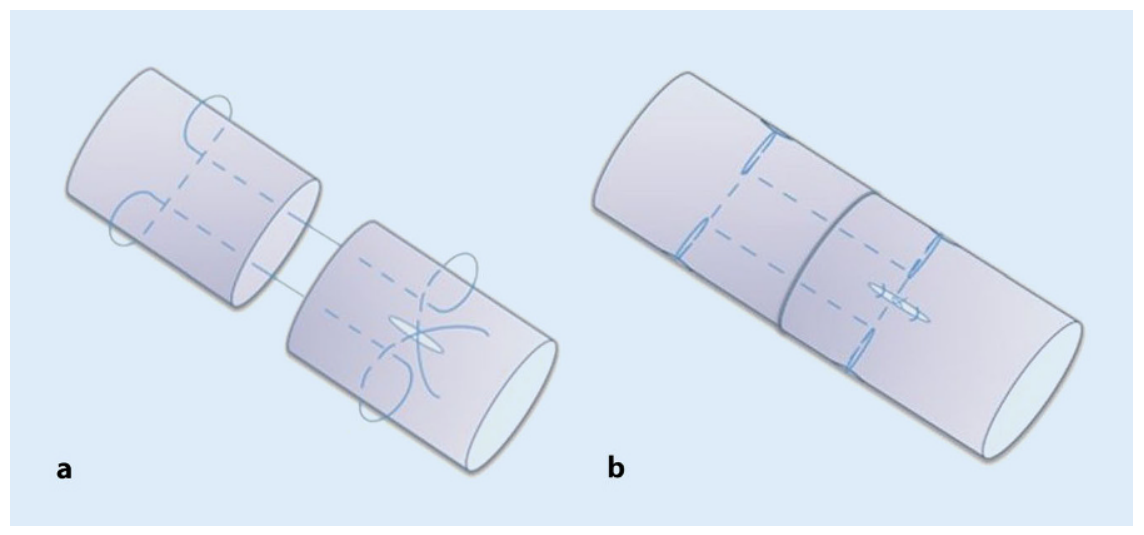

Abb. 6 \ Zweistrangtechniknach Kirchmayr-Kessler. in der Modifikation nach Zechner. a Anordnung der Nahttechnik, b Versenken des Knotens durch Längsinzision der Sehne

konnte die Analyse von Rupturraten in klinischen Studien der letzten 20 Jahre keinen signifikanten Unterschied zwischen Zweistrang- und Mehrstrangtechniken zeigen $[24,25]$. Auch bezüglich der Nachbehandlung (aktiv oder passiv) konnte in Übersichtsarbeiten kein statistisch signifikanter Unterschied in den Gesamtkomplikationen gefunden werden $[2,19]$. Unter der passiven Nachbehandlung betrug die Gesamtkomplikationsrate $13 \%$ mit $4 \%$ Rupturen und $9 \%$ Bewegungseinschränkungen der betroffenen Finger. Bei aktiver Nachbehandlung lag die Gesamtkomplikationsrate bei $11 \%$ mit $5 \%$ Rupturen und $6 \%$ Bewegungseinschränkungen [19]. Somit hatten passive Nachbehandlungsprotokolle eine statistisch signifikant niedrigere Rupturrate bei höherem Risiko einer Bewegungseinschränkung verglichen mit einer aktiven Nachbehandlung [19]. Bei aktiver Nachbehandlung fand sich kein statistisch signifikanter Unterschied bezüglich der Rupturrate bei Zweistrang- oder Mehrstrangtechniken [24]. Auch hinsichtlich des funktionellen Ergebnisses konnte für Mehrstrangtechniken keine Überlegenheit in klinischen Studien gezeigt werden [6]. Interessant ist jedoch, dass aktuell ein Trend zur Vereinfachung dieser komplexen Nahttechniken zu beobachten ist [24]. Das vermehrte Einbringen von Nahtmaterial in die Sehne vermag nicht nur die Gleitfähigkeit zu beeinflussen, sondern auch störende Effekte auf die Tenozytenaktivität und somit auf die Sehnenheilung zu haben [12, 24, 25]. So könnte es sein, dass die biomechanisch vermeintlich

\section{Epitendinöse Naht}

Eine zirkulär epitendinöse Naht dient dazu, minimale Lücken nach erfolgter Kernnaht zu schließen, um eine glatte Adaptation zu erreichen [24]. Als essenzielle Komponente der Sehnennaht trägt sie rund $10-50 \%$ zu der Gesamtstabilität bei [8]. Auch hier stehen diverse Nahttechniken zur Verfügung, wie z.B. die einfach fortlaufende Naht nach Kleinert oder Kreuzstichnähte nach Lembert und Halsted [10, 27]. Trotz der biomechanischen Überlegenheit anderer Nähte wird die fortlaufende Ringnaht aufgrund ihrer Einfachheit sehr häufig verwendet [27, 29]. Entsprechend den beschriebenen Veränderungen in der unmittelbaren Traumazone soll die Naht mindestens $2 \mathrm{~mm}$ davon entfernt gesetzt werden [8, 27].

\section{Nahtmaterial}

Die Suche nach dem perfekten Nahtmaterial gestaltet sich ebenso schwierig wie jene nach der optimalen Nahttechnik. Trotz einer Vielzahl an biomechanischen Untersuchungen gibt es keinen einheitlichen Konsens darüber [24]. Die Auswahl beruht vielmehr auf der individuellen Erfahrung des Operateurs bzw. der jeweiligen Verfügbarkeit des Nahtmaterials. Neben der Reißfestigkeit sind v. a. eine geringe Reaktion mit dem umliegenden Sehnengewebe und einfache Handhabung zu beachten [29]. Den häufigsten Einsatz finden nicht resorbierbare synthetische Nahtmaterialien, die eine gute Gleitfähigkeit aufweisen (z. B., Supramid ${ }^{\otimes}$ [B. Braun AG, Melsungen, Deutschland], Ethibond ${ }^{\circledast}$, Ethilon ${ }^{\circledR}$, Prolene $^{\circledast}$ [alle drei: Ethicon-Germany / Johnson \& Johnson Medical GmbH, Norderstedt, Deutschland]). Auch resorbierbare Fäden mit ausreichender Resorptionszeit (z.B. PDS ${ }^{\circledast}$ [Ethicon], Maxon $^{\circledR}$ [Medtronic, Minneapolis, MN, USA]) eignen sich gut für Sehnennähte. Eine sehr hohe Stabilität bietet der FiberWire $^{\circledR}$ (Arthrex, Naples, FL, USA) jedoch ist hier die geringere Knotensicherheit zu beachten [29].

Die Fadenstärke für die Kernnaht liegt in der Regel zwischen 3/0 und 4/0, wobei hinsichtlich der Rupturrate kein Un- 
terschied zwischen einem 3/0- und 4/0Faden aufgezeigt werden konnte [6]. Für Kernnähte nicht zu empfehlen sind Fäden der Stärke 5/0 [29]. Diese eignen sich jedoch sehr gut für epitendinöse Adaptationsnähte, wofür auch Fäden der Stärke 6/0 häufigen Einsatz finden.

\section{Fazit für die Praxis}

\section{- Entsprechend der International Federation of Societies for Surgery of the Hand (IFSSH) liegt aktuell kein Goldstandard hinsichtlich der idealen Nahttechnik oder des optimalen Nahtmaterials vor. \\ - In groß angelegten Studien wurde hinsichtlich der Rupturrate und des funktionellen Ergebnisses kein statis- tisch signifikanter Unterschied zwi- schen Zweistrang- und Mehrstrang- techniken nachgewiesen. \\ - Die aktive Nachbehandlung führt ge- genüber der passiven zu geringfügig besseren funktionellen Ergebnissen auf Kosten einer leicht erhöhten Rup- turrate unabhängig von der Anzahl der Kernnähte. \\ - Die Wahl zwischen einer Zweistrang- oder Mehrstrangnaht sowie dem postoperativen Nachbehandlungs- konzept obliegt dem Operateur. \\ - Um ein zufriedenstellendes funk- tionelles Ergebnis zu erhalten, ist die frühzeitige aktive oder passive Nachbehandlung unerlässlich.}

\section{Korrespondenzadresse}

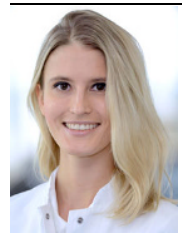

Dr. med. univ. I. A. Ederer Klinik für Hand-, Plastische, Rekonstruktive und Verbrennungschirurgie, BG Klinik Tübingen Schnarrenbergstr. 95, 72076 Tübingen, Deutschland iederer@bgu-tuebingen.de

\section{Einhaltung ethischer Richtlinien}

Interessenkonflikt. I.A. Ederer, A. Nusche, A. Daigeler und C. Bösch geben an, dass kein Interessenkonflikt besteht.
Dieser Beitrag beinhaltet keine von den Autoren durchgeführten Studien an Menschen oder Tieren.

The supplement containing this article is not sponsored by industry.

\section{Literatur}

1. Azzi AJ, Aldekhayel S, Boehm KS, Zadeh T (2017) Tendon rupture and tenosynovitis following internal fixation of distal radius fractures. Plast Reconstr Surg 139:717e-724e

2. Chesney A, Chauhan A, Kattan A et al (2011) Systematic review of flexor tendon rehabilitation protocols in zone ll of the hand. Plast Reconstr Surg 127:1583-1592

3. Clayton RAE, Court-Brown CM (2008) The epidemiology of musculoskeletal tendinous and ligamentous injuries. Injury 39:1338-1344

4. Esenwein P, Sonderegger J, Gruenert J et al (2013) Complications following palmar plate fixation of distal radius fractures: a review of 665 cases. Arch Orthop Trauma Surg 133:1155-1162

5. Hahn P, Unglaub F, Spies CK (2015) Diagnostik der Beugesehnenverletzungen der Hand. Orthopäde 44:743-747

6. Hardwicke JT, Tan JJ, Foster MA, Titley OG (2014) A systematic review of 2-strand versus multistrand core suture techniques and functional outcome after digital flexor tendon repair. J Hand Surg Am 39:686-695

7. de Jong JP, Nguyen JT, Sonnema AJM et al (2014) The incidence of acute traumatic tendon injuries in the hand and wrist: a 10-year population-based study. Clin Orthop Surg 6:196-202

8. Kamal RN, Yao J (2017) Evidence-based medicine Plast Reconstr Surg 140:130e-139e

9. Langer M, Surke C, Wieskötter B (2011) Prinzipien der Sehnenbehandlung: Beugesehnen. In: Towfigh $\mathrm{H}$, Hierner R, Langer M, Friedel R (Hrsg) Handchirurgie, 2. Aufl. Springer, Berlin, S102-138

10. Langer MF, Oeckenpöhler S, Kösters $C$ et al (2015) Nahttechniken der Beugesehnen der Hand. Orthopäde 44:748-756

11. Lögters TT, Windolf J (2015) Flexor tendon injuries. OPJ31:160-166

12. Momose T, Amadio PC, Zhao C et al (2000) The effect of knot location, suture material, and suture size on the gliding resistance of flexor tendons. JBiomed Mater Res 53:806-811

13. Moriya K, Yoshizu T, Tsubokawa N et al (2016) Outcomes of release of the entire A4 pulley after flexor tendon repairs in zone $2 \mathrm{~A}$ followed by early active mobilization. J Hand Surg Eur Vol 41:400-405

14. Niwa T, Uchiyama S, Yamazaki Het al (2010) Closed tendon rupture as a result of Kienböck disease. JPlast Surg Hand Surg 44:59-63

15. PruittDL, Tanaka H, Aoki M, ManskePR (1996)Cyclic stress testing after in vivo healing of canine flexor tendon lacerations. J Hand Surg Am 21:974-977

16. Rudigier J, Meier R (2014) Beugesehnenverletzungen. In: Kurzgefasste Handchirurgie Klinik und Praxis, 6. Aufl. Thieme, Stuttgart, S 192-209

17. Schünke M, Schulte $E$, Schumacher U (2009) Obere Extremität - Topographie der Muskulatur. In: Prometheus - LernAtlas der Anatomie: Allg. Anatomie und Bewegungssystem, 2. Aufl. Thieme, Stuttgart, S324-347

18. Schweizer A. Das A2 Ringband und die Beugesehnenscheide. http://www.turntillburn.ch/cms/ ttb/index.php?id=93\&L=2\%7C0. Zugegriffen: 03 . Febr. 2018
19. Starr HM, Snoddy M, Hammond KE, Seiler JG (2013) Flexor tendon repair rehabilitation protocols: a systematic review. J Hand Surg Am 38:1712-1717

20. Strickland JW (1995) Flexor tendon injuries: I. foundations of treatment. J Am Acad Orthop Surg 3:44-54

21. Tan J, Tang JB (2004) Locking repairs for obliquely cut tendons: effects of suture purchase and directions of locking circles. J Hand Surg Am 29:891-897

22. Tan JS, Oh L, Louis DS (2009) Variations of the flexor digitorum superficialis as determined by an expanded clinical examination. J Hand Surg Am 34:900-906

23. Tang JB (2014) Release of the A4 pulley to facilitate zone II flexor tendon repair. J Hand Surg Am 39:2300-2307

24. The IFSSH Scientific Committee on Flexor Tendon Repair (2015) Trends in flexor tendon surgery over the last 10 years. IFSSH Ezine 5:21-34. http://ifssh. info/ezine.html.Zugegriffen:5. Febr. 2018

25. Thurman RT, Trumble TE, Hanel DP et al (1998) Two-, four-, and six-strand zone II flexor tendon repairs: an in situ biomechanical comparison using a cadaver model. J Hand Surg Am 23:261-265

26. Verdan CE (1960) Primary repair of flexor tendons. JBone Joint Surg Am 42-A:647-657

27. Wieskötter B, Herbort M, Langer M et al (2018) The impact of different peripheral suture techniques on the biomechanical stability in flexor tendon repair. Arch Orthop Trauma Surg 138:139-145

28. Winkelman NZ (1983) An accessory flexor digitorum profundus indicis. J Hand Surg Am 8:70-71

29. Wu YF, Tang JB (2014) Recent developments in flexor tendon repair techniques and factors influencing strength of the tendon repair. J Hand Surg Eur Vol 39:6-19

30. Zechner W, Buck-Gramcko D, Lohmann $\mathrm{H}$ et al (1985) Überlegungen zur Verbesserung der Nahttechnik bei Beugesehnenverletzungen. Klinische und Experimentelle Studie. Handchir Mikrochir Plast Chir 17:8-13 